\title{
Acute Effects of Aerobic Exercise on Muscle Strength and Power in Trained Male Individuals: A Systematic Review with Meta-analysis
}

\author{
Adrian Markov ${ }^{1} \cdot$ Helmi Chaabene $^{4} \cdot$ Lukas Hauser $^{1} \cdot$ Sebastian Behm $^{1} \cdot$ Wilhelm Bloch $^{2} \cdot$ Christian Puta $^{3}$. \\ Urs Granacher ${ }^{1}$ (D)
}

Accepted: 26 November 2021 / Published online: 8 December 2021

(c) The Author(s) 2021

\begin{abstract}
Background Concurrent training can be an effective and time-efficient method to improve both muscle strength and aerobic capacity. A major challenge with concurrent training is how to adequately combine and sequence strength exercise and aerobic exercise to avoid interference effects. This is particularly relevant for athletes.

Objective We aimed to examine the acute effects of aerobic exercise on subsequent measures of muscle strength and power in trained male individuals.

Design We performed a systematic review with meta-analysis.

Data Sources Systematic literature searches in the electronic databases PubMed, Web of Science, and Google Scholar were conducted up to July 2021.

Eligibility Criteria for Selecting Studies Studies were included that applied a within-group repeated-measures design and examined the acute effects of aerobic exercise (i.e., running, cycling exercise) on subsequent measures of lower limb muscle strength (e.g., maximal isometric force of the knee extensors) and/or proxies of lower limb muscle power (e.g., countermovement jump height) in trained individuals.

Results Fifteen studies met the inclusion criteria. Aerobic exercise resulted in moderate declines in muscle strength (standardized mean difference $[\mathrm{SMD}]=0.79 ; p=0.003)$. Low-intensity aerobic exercise did not moderate effects on muscle strength $(\mathrm{SMD}=0.65 ; p=0.157)$ while moderate-to-high intensity aerobic exercise resulted in moderate declines in muscle strength $(\mathrm{SMD}=0.65 ; p=0.020)$. However, the difference between subgroups was not statistically significant $(p=0.979)$. Regarding aerobic exercise duration, large declines in muscle strength were found after $>30$ min $(\mathrm{SMD}=1.02 ; p=0.049)$ while $\leq 30 \mathrm{~min}$ of aerobic exercise induced moderate declines in muscle strength $(\mathrm{SMD}=0.59 ; p=0.013)$. The subgroup difference was not statistically significant $(p=0.204)$. Cycling exercise resulted in significantly larger decrements in muscle strength $(\mathrm{SMD}=0.79 ; p=0.002)$ compared with running $(\mathrm{SMD}=0.28 ; p=0.035)$. The difference between subgroups was statistically significant $(p<0.0001)$. For muscle power, aerobic exercise did not result in any statistically significant changes $(\mathrm{SMD}=0.04 ; p=0.846)$.

Conclusions Aerobic exercise induced moderate declines in measures of muscle strength with no statistically significant effects on proxies of muscle power in trained male individuals. It appears that higher compared with lower intensity as well as longer compared with shorter aerobic exercise duration exacerbate acute declines in muscle strength. Our results provide evidence for acute interference effects when aerobic exercies is performed before strength exercises. These findings may help practitioners to better prescribe single training sessions, particularly if environmental and/or infrastructural reasons (e.g., availability of training facilities) do not allow the application of strength training before aerobic exercise.
\end{abstract}

Urs Granacher

urs.granacher@uni-potsdam.de

Extended author information available on the last page of the article 


\section{Key Points}

Aerobic exercise resulted in acute moderate declines in measures of muscle strength but not power in trained male individuals.

There was a negative influence of prior moderate-to-high intensity, as well as longer aerobic exercise durations (i.e., $>30 \mathrm{~min}$ ) on muscle strength in trained male individuals. Low-intensity and short duration (i.e., $\leq 30 \mathrm{~min}$ ) aerobic exercise appears not to compromise strength performance.

Cycling compared with running exercise causes larger decrements in lower limb muscle strength.

\section{Introduction}

Many sports require the simultaneous development of muscle strength and aerobic capacity to successfully perform in competition [1-3]. Concurrent training (CT), which is the combination of strength and endurance training, can be an effective and time-efficient method to improve both muscle strength and aerobic capacity $[4,5]$. The long-term effects of CT on measures of muscle strength and muscle power are well established in the literature [6-10]. A major challenge within $\mathrm{CT}$ is how to adequately combine strength and endurance training to avoid interference effects [11-13]. In this context, interference has been reported if $\mathrm{CT}$ was contrasted with single-mode strength training. The results showed that the adaptive potential of CT to improve muscle strength is attenuated compared with single-mode strength training [7, 9, 13-15]. In general, the interference may occur in elite and recreational athletes if they exercise at high training volumes. However, the training volume of recreational athletes is usually low to moderate, which prevents interference if the training is appropriately prescribed. In contrast, elite athletes in sports that demand both high levels of muscle strength and aerobic capacity exercise up to $25 \mathrm{~h}$ per week [16], which increases the likelihood of experiencing interference effects due to timely proximity of strength and endurance training.

This raises the question with regard to the most effective CT sequence to minimize interference. Murlasits and colleagues [17] conducted a systematic review with metaanalysis and examined the long-term effects of intra-session exercise sequence during CT on lower body muscle strength and maximal aerobic capacity in healthy individuals aged 14-66 years. These authors reported that the sequence strength exercises (SE) before aerobic exercises (AE) is more effective to improve muscle strength than AE before SE. Using a similar meta-analytical approach, Eddens and colleagues [18] confirmed the findings of Murlasits et al. [17] for lower limb maximal strength in healthy individuals aged $18-65$ years.

While the long-term effects of intra-session exercise sequencing during CT on muscle strength are well established, little is known on the acute effects of exercise sequencing during CT. In this context, the term 'acute' refers to the impact of the sequence (SE before $\mathrm{AE}$ or vice versa) on muscle strength and power after a single training session. This is of particular relevance for elite athletes who realize up to four training sessions per day [16]. As such, it is not always possible to follow the recommended SE-AE sequence $[17,18]$ in every single training session because of the training schedule or the availability of training facilities.

Of note, the available studies on the acute effects of $\mathrm{AE}$ on measures of muscle strength and power have shown inconsistent findings. More specifically, while some studies reported attenuated strength and power outcomes [19-21], others showed post-activation performance enhancement (PAPE) most likely triggered by the previous AE [22-24]. For example, Lepers et al. [25] studied the acute effects of two different cycling modalities (i.e., constant and variable power output) on measures of muscle strength in trained male triathletes aged 33 years. Training was conducted at $\sim 63-86 \%$ of the maximal aerobic power (i.e., highest power in Watts across 2 min during a continuous incremental cycling test until exhaustion) for $30 \mathrm{~min}$. The results revealed a decrease in knee extensor isometric maximal voluntary contraction (IMVC) [ $\Delta 11 \%$ ] following both cycling modalities. In another study, Wilhem and colleagues [26] examined the effects of $30 \mathrm{~min}$ of cycling or running at $75-85 \%$ of the respiratory compensation point on measures of muscle strength and power in recreationally trained male individuals aged 23 years. These authors could not find any significant performance changes after both protocols. Additionally, in trained long-distance runners aged 24 years, Vuorimaa and colleagues [27] reported improved vertical jump performance $(\Delta 10-15 \%)$ following $40 \mathrm{~min}$ of continuous or intermittent (i.e., 2-min run/2-min rest) running at $80 \%$ and $100 \%$ of the maximum running speed achieved during a graded maximal oxygen consumption $\left(V \mathrm{O}_{2 \max }\right)$ test, respectively. The observed discrepancy in the literature is most likely due to differences in the participants' training status [12], the type of task used to quantify fatigue [12], AE-related neuromuscular fatigue [28, 29], and/or PAPE [30]. Neuromuscular fatigue appears to be a major candidate responsible for the observed AE-related performance declines [31-33]. For this systematic review, we have defined neuromuscular fatigue as a reduction in muscle force and power in response to sustained contractile activity $[28,29]$. There is evidence that 
$\mathrm{AE}$ intensity and volume (i.e., duration) are key moderators for AE-induced neuromuscular fatigue [34, 35]. In contrast, PAPE refers to gains in muscle power, speed, and maximal strength following conditioning contractions [30]. Other important factors that might (partly) explain the conflicting findings in the literature are the complexity of the underpinning mechanisms of adaptations as well as factors such as the rest between AE and SE and type of SE/AE [4, 12, 36].

Accordingly, it seems necessary to perform a systematic review of the literature on the acute effects of AE on measures of muscle strength and power in trained individuals. Therefore, this systematic review with meta-analysis aimed at (1) examining the acute effects of $\mathrm{AE}$ on subsequent measures of muscle strength and power in trained male individuals and (2) investigating the influence of potential moderating factors such as $\mathrm{AE}$ intensity, duration, and type (i.e., low vs moderate-to-high intensity $\mathrm{AE}, \leq 30 \mathrm{~min}$ vs $>30 \mathrm{~min}$ $\mathrm{AE}$, and cycling vs running exercise) on muscle strength and power. With reference to the relevant literature [19-21, 25], we hypothesized that findings from the included AE studies would show declines in measures of muscle strength and power due to AE-related neuromuscular fatigue. We further expected that the magnitude of declines in measures of muscle strength and power would be moderated by different exercise modalities (e.g., AE intensity and duration) [26, 37] and types (i.e., cycling vs running) [12].

\section{Methods}

This meta-analysis was conducted according to the Preferred Recording Items for Systematic Review and Meta-analysis (PRISMA) statements [38].

\subsection{Systematic Literature Search}

A systematic literature search was conducted in the electronic databases PubMed, Web of Science, and Google Scholar up to July 2021. Keywords were collected through expert opinion, literature review, and controlled vocabulary (e.g., Medical Subject Headings [MeSH]). The following Boolean search syntax is an example of a PubMed search: "(strength OR resistance OR endurance OR aerobic OR concurrent OR running OR cycling OR rowing OR swimming) AND (training OR exercise) AND (order OR intra-session OR sequence OR within-session OR "same day" OR "separate day" OR acute OR short-term) AND (combined OR combination OR additional OR subsequent) NOT (patients OR elderly OR cancer OR diabetes OR injury OR protein OR stretching OR obese OR blood OR diet OR rat OR cognitive OR stroke)". Where applicable, filters were applied for the type of article (e.g., no reviews). The search results were independently screened for titles, abstracts, and full texts by two authors (AM, SB). In addition, reference lists of the included studies were screened for more compatible studies. An overview of the search process is displayed in Fig. 1.

\subsection{Inclusion and Exclusion Criteria}

A PICOS (participants, intervention, comparator, study outcomes, and study design) approach was used to rate studies for eligibility [38]. Specifically, the following inclusion criteria were defined a priori: (1) participants: trained youth and young adults (2) intervention: AE (i.e., running, cycling) (3) comparator: post-test measure within $\leq 15$ min after AE (4) study outcomes: muscle strength (e.g., maximal isometric force of the knee extensors) and/or power, (e.g., countermovement jump height), and (5) study design: within-group repeated-measures design. Of note, there is no well-accepted definition of the term "trained," especially in the context of CT [39]. However, with reference to the included studies and based on the definition provided by MacMahon and Parrington [40], we consider trained individuals as athletes actively engaging in sports training, where the main motivation

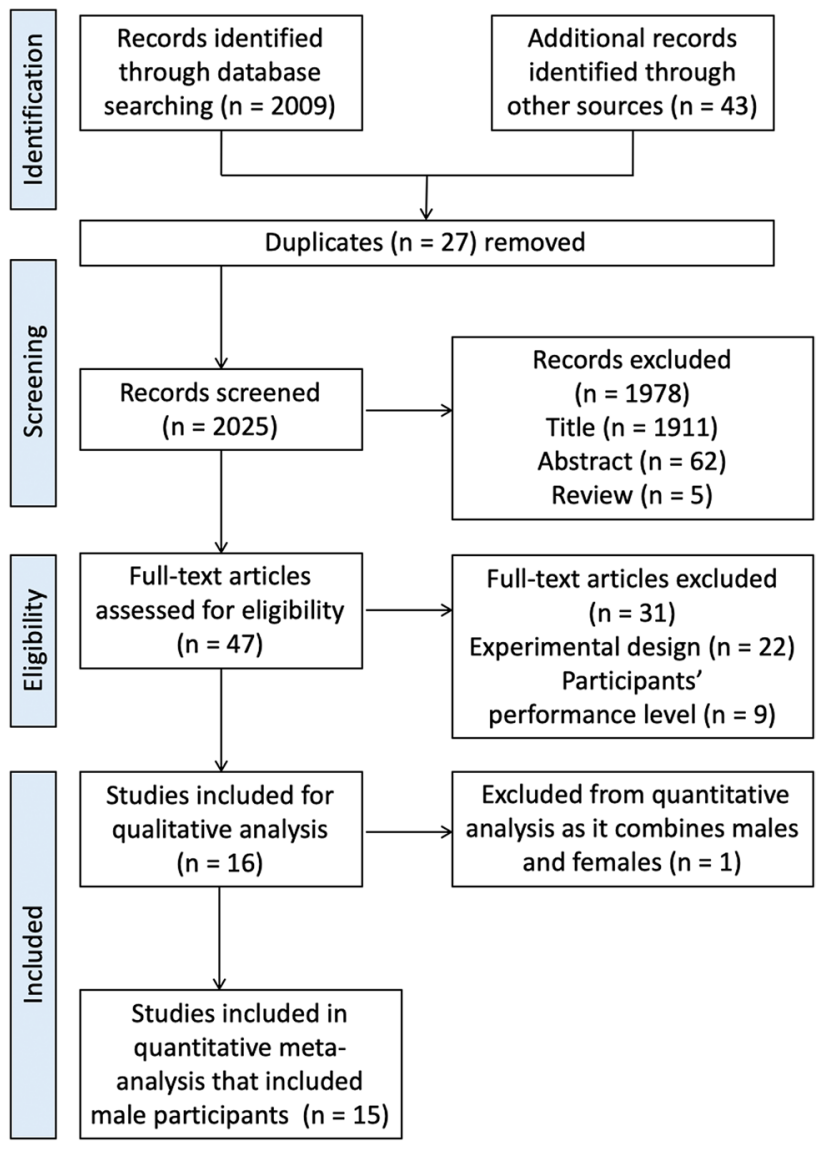

Fig. 1 Flow chart illustrating the search and selection process of this systematic review 
or goal is to improve sport-specific skills, performance, or results (technical, physical, or tactical) for competition. In this context, AE is an umbrella term for all types of physical exercise to improve cardiorespiratory fitness (e.g., oxygen transport and utilization systems) [41]. We excluded studies involving individuals with pre-existing health problems (e.g., diabetes mellitus, hypertension) or with no training background, lack of pre-AE and post-AE measures, and studies not written in English.

\subsection{Data Extraction}

Studies were coded for the variables displayed in Table 1. When multiple tests were used for the same outcome measure, the most accurate and frequently used protocols were selected based on expert opinion (AM, HC, LH, and UG) (Table 1). Two authors (AM and SB) independently extracted data from the included studies in a standardized template created with Microsoft Excel (version 16.16.27). In cases of disagreement regarding data extraction and study eligibility, another co-author (HC) was consulted for clarification. To compute effect sizes, pre-test and post-test means and standard deviations for measures of muscle strength and power were used. The characteristics of the included studies are displayed in Table 2. Of note, intermittent and continuous AE protocols were treated in the same way in terms of duration. More specifically, the cumulative duration of the work intervals, including the respective recovery periods between intervals, were considered for intermittent protocols. In the case of missing data, authors were contacted and kindly asked to provide the data. If the authors did not respond and the data were displayed graphically, we used a software tool (GetData Graph Digitizer; http://www.getdata-graph-digitizer.com) [42], which has been shown to be accurate and precise [43], to extract data from graphs.

Table 1 Testing protocols across the different measures of physical fitness considered for statistical analyses

\begin{tabular}{ll}
\hline Outcome categories & Ranking \\
\hline Muscle strength & $\bullet$ Maximal isometric force \\
& of the knee extensors \\
$\bullet$ Maximal isokinetic & torque of the knee exten- \\
& sors \\
& $\bullet$ One-maximum repetition \\
& knee extensors (leg \\
& press) \\
& $\bullet$ CMJ height \\
Muscle power & $\bullet$ CMJ peak power \\
&
\end{tabular}

$C M J$ counter movement jump

\subsection{Risk of Bias Assessment}

The methodological quality of the included studies is summarized in Table 2. The risk of bias assessment was conducted independently by two authors (AM, LH), using the quality appraisal tool developed by Galna et al. [44]. The applied quality appraisal tool focuses on internal and external validity as well as the reproducibility of the study. It includes 14 items and allows a rating between 0 and 1 , with 1 representing the maximum value for each of the items. There is no particular classification (e.g., low, acceptable, high) foreseen with this rating system. The higher the total score, the better the quality of the respective study. Of note, the highest possible score is 14 . Total scores ranged between 7 and 11 (Table 2). In the case of disagreement, another co-author (HC) was consulted for clarification.

\subsection{Statistical Analyses}

The effects of $\mathrm{AE}$ on subsequent measures of muscle strength and power were examined by calculating standardized mean differences (SMDs) for pre-AE and post-AE measures of the respective studies. To estimate the overall effect of $\mathrm{AE}$ on subsequent measures of muscle strength and power, we pooled effect sizes using a random-effects pooling model approach with the Sidik-Jonkman estimator method and Hartung-Knapp adjustment [45] using the packages "meta" [46] and "metafor" [47]. In addition, independent subgroup analyses were calculated for the exercise modalities AE intensity (low vs moderate to high), AE duration ( $\leq 30 \mathrm{~min}$ vs $>30 \mathrm{~min}$ ), and $\mathrm{AE}$ type (running vs cycling exercise). Of note, because of a limited number of studies per group, subgroup analyses were calculated only for measures of muscle strength and not power. Based on the guidelines proposed by the American College of Sports Medicine [48], we classified AE intensity as low $(<70 \%$ $V \mathrm{O}_{2 \max }$; < critical power; $<$ peak aerobic power; $<$ anaerobic or lactate threshold), or moderate to high $\left(>70 \% V_{2} \mathrm{O}_{2 \max }\right.$ or maximal heart rate; $>$ critical power; $>$ anaerobic or lactate threshold; rate of perceived exertion $\geq 15$ ). Critical power was defined as the highest power output in Watts that can be sustained for a given period of time [49]. Standardized mean differences were interpreted according to Cohen [50] as "trivial" $(<0.2)$, "small" $(0.2 \leq \mathrm{SMD}<0.5)$, "moderate" $(0.5 \leq \mathrm{SMD}<0.8)$, or "large" $(\mathrm{SMD} \geq 0.8) . I^{2}$ statistics were used to examine between-study heterogeneity. According to Higgins et al. [51], heterogeneity in the form of $I^{2}$ statistics was interpreted as "low" (25\%), "moderate" (50\%), and "high" (75\%). The level of significance was set at $p<0.05$. All analyses were conducted using R (version 4.0.2, 2020) [52] and validated open-source packages [47]. 


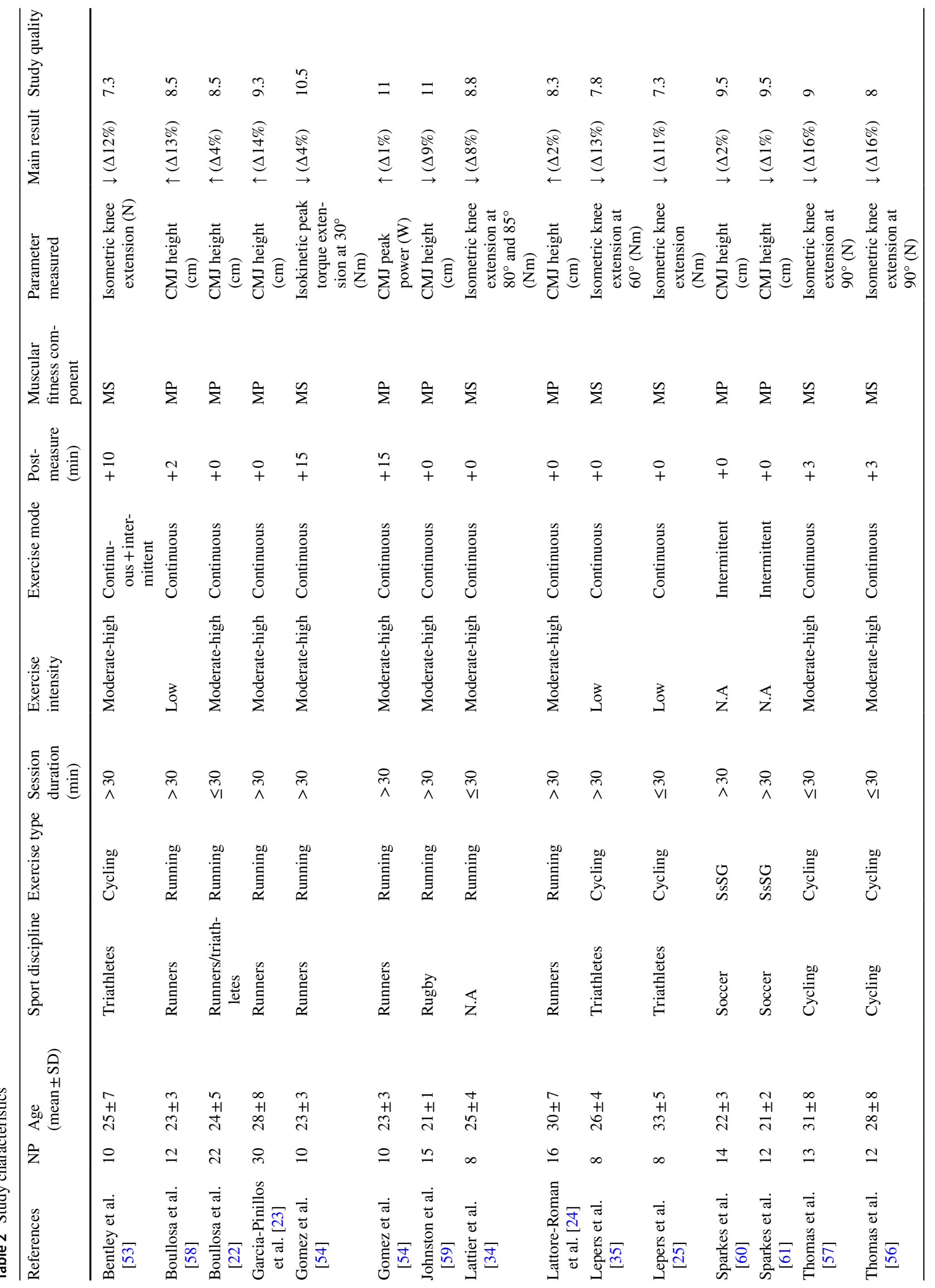




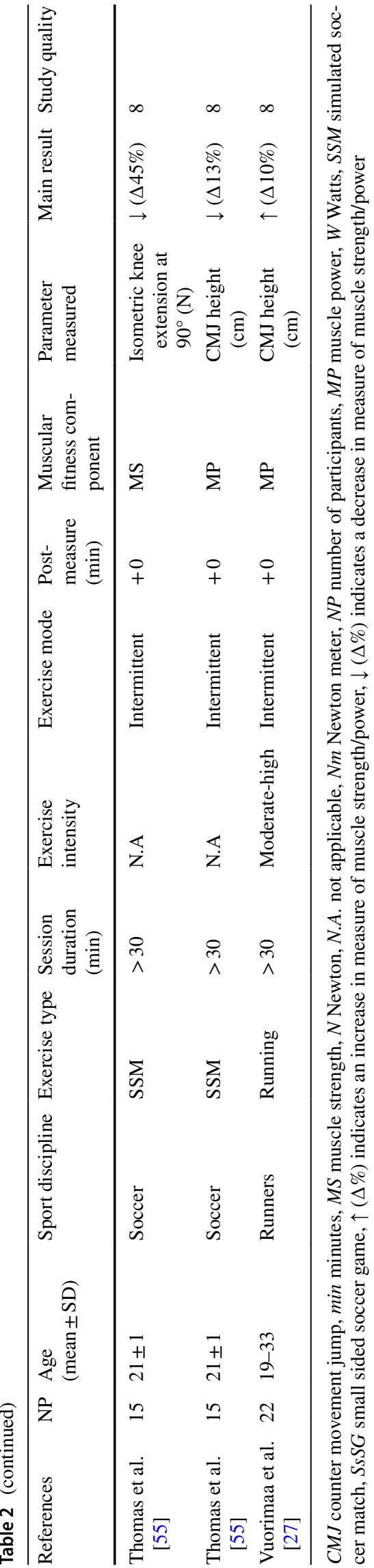

\section{Results}

\subsection{Study Characteristics}

Our systematic search identified 2052 potential articles (Fig. 1). After screening for titles, abstracts, and full texts, 15 studies were considered eligible with a total of 197 participants. The age range within the included studies was 18-42 years. Eight studies examined AE effects on measures of muscle strength [25, 34, 35, 53-57], and another nine studies reported AE effects on proxies of muscle power [23, 24, 27, 54, 55, 58-61]. Running-based AE was examined in seven studies [23, 24, 27, 34, 54, 58, 59] while cycling exercises were applied in five studies [25, 35, 53, 56, 57]. Three studies scrutinized the effects of sport-specific AE (i.e., small-sided soccer games) [55, 60, 61]. Likewise, two studies applied low-intensity AE [25, 35] while 11 studies used moderate-to-high intensity AE protocols [23, 24, 27, $34,53,54,56,57,59,62,63]$. However, three studies [55, $60,61]$ did not report any information on exercise intensity. In terms of AE duration, four studies used protocols lasting $\leq 30 \mathrm{~min}[25,34,56,57]$ and 11 studies applied protocols $>30$ min [23, 24, 27, 35, 53-55, 58-61]. Originally 16 studies matched the inclusion criteria. Because of the fact that only one paper considered male and female individuals [22], this study was excluded for the quantitative metaanalysis to ensure consistency throughout the data.

\subsection{Acute Effects of Aerobic Exercise on Measures of Muscle Strength and Power}

The overall acute effects of AE on measures of lower limb muscle strength and power are illustrated in Figs. 2 and 3. Aerobic exercise resulted in moderate declines in measures of muscle strength (SMD $=0.79$ [95\% confidence interval (CI) 0.38 to 1.21 ]; $p=0.003 ; I^{2}=15 \%$, eight studies, Fig. 2 ). Regarding muscle power, $\mathrm{AE}$ did not produce any statistically significant changes $(\mathrm{SMD}=0.04[95 \% \mathrm{CI}-0.39$ to $0.46] ; p=0.846 ; I^{2}=60 \%$, nine studies, Fig. 3).

\subsection{Results of the Subgroup Analyses for Measures of Muscle Strength}

Results of the subgroup analyses are displayed in Table 3. Low-intensity AE did not cause statistically significant effects on muscle strength (SMD $=0.65[95 \% \mathrm{CI}-1.45$ to 2.75], $p=0.157 ; I^{2}=0 \%$, two studies) while moderate-to-high intensity $\mathrm{AE}$ resulted in moderate declines in muscle strength (SMD $=0.65$ [95\% CI 0.16-1.13], $p=0.020 ; I^{2}=0 \%$, five studies). However, the difference between subgroups was not statistically significant $(p=0.979)$. Regarding AE duration, 


\begin{tabular}{|c|c|c|c|c|c|c|c|c|}
\hline & Pre & & & Post & & & & Std. Mean Difference \\
\hline Study & Mean & SD & Total & Mean & SD & Total & Weight & IV, Random, 95\% Cl \\
\hline Bentley et al. [53] & 331.00 & 29.00 & 10 & 292.00 & 31.00 & 10 & $11.5 \%$ & $1.24[0.27 ; 2.22]$ \\
\hline Gomez et al. [54] & 246.20 & 30.10 & 10 & 237.40 & 34.10 & 10 & $13.1 \%$ & $0.26[-0.62 ; 1.14]$ \\
\hline Lattier et al. [34] & 298.70 & 92.60 & 8 & 273.20 & 70.40 & 8 & $11.3 \%$ & $0.29[-0.69 ; 1.28]$ \\
\hline Leppers et al. [35] & 235.00 & 37.00 & 8 & 206.00 & 29.00 & 8 & $10.6 \%$ & $0.82[-0.21 ; 1.86]$ \\
\hline Leppers et al. [25] & 253.00 & 60.00 & 8 & 230.20 & 14.40 & 8 & $11.1 \%$ & $0.49[-0.51 ; 1.49]$ \\
\hline Thomas et al. [57] & 536.00 & 143.00 & 13 & 452.00 & 121.00 & 13 & $14.9 \%$ & $0.61[-018 ; 1.40]$ \\
\hline Thomas et al. [56] & 579.10 & 110.10 & 12 & 485.80 & 102.20 & 12 & $13.8 \%$ & $0.85[0.01 ; 1.69]$ \\
\hline Thomas et al. [55] & 464.10 & 107.30 & 15 & 256.30 & 128.10 & 15 & $13.6 \%$ & $1.71[0.86 ; 2.56]$ \\
\hline Total $(95 \% \mathrm{Cl})$ & & & 84 & & & 84 & $100 \%$ & $0.79[0.38 ; 1.21]$ \\
\hline
\end{tabular}

Fig. 2 Forest plot for the overall effect of aerobic exercise on subsequent measures of muscle strength

\begin{tabular}{|c|c|c|c|c|c|c|c|c|}
\hline & Pre & & & Post & & & & Std. Mean Difference \\
\hline Study & Mean & SD & Total & Mean & SD & Total & Weight & IV, Random, 95\% Cl \\
\hline Boullosa et al. [58] & 30.30 & 4.70 & 12 & 31.30 & 4.70 & 12 & $10.2 \%$ & $-0.21[-1.01 ; 0.60]$ \\
\hline Gomez et al. [54] & 3149.80 & 505.10 & 10 & 3195.00 & 374.60 & 10 & $9.3 \%$ & $-0.10[-0.97 ; 0.78]$ \\
\hline Johnston et al. [59] & 39.00 & 6.00 & 15 & 35.00 & 7.00 & 15 & $11.0 \%$ & $0.60[-0.14 ; 1.33]$ \\
\hline Garcia-P. et al. [23] & 38.00 & 5.00 & 30 & 41.00 & 5.00 & 30 & $13.9 \%$ & $-0.59[-1.11 ;-0.07]$ \\
\hline Lattore-R. et al. [24] & 38.63 & 4.87 & 16 & 39.45 & 4.95 & 16 & $11.5 \%$ & $-0.16[-0.86 ; 0.53]$ \\
\hline Sparkes et al. [60] & 29.60 & 10.10 & 14 & 25.70 & 9.30 & 14 & $10.8 \%$ & $0.39[-036 ; 1.14]$ \\
\hline Sparkes et al. [61] & 25.20 & 9.50 & 12 & 21.80 & 13.20 & 12 & $10.1 \%$ & $0.29[-0.52 ; 1.09]$ \\
\hline Thomas et al. [55] & 38.70 & 4.30 & 15 & 33.90 & 5.00 & 15 & $10.6 \%$ & $1.00[0.24 ; 1.77]$ \\
\hline Vuorimaa et al. [27] & 31.30 & 5.50 & 22 & 34.50 & 5.10 & 22 & $12.7 \%$ & $-0.59[-1.20 ; 0.001]$ \\
\hline Total $(95 \% \mathrm{Cl})$ & & & 146 & & & 146 & $100 \%$ & $0.04[-0.39 ; 0.46]$ \\
\hline
\end{tabular}

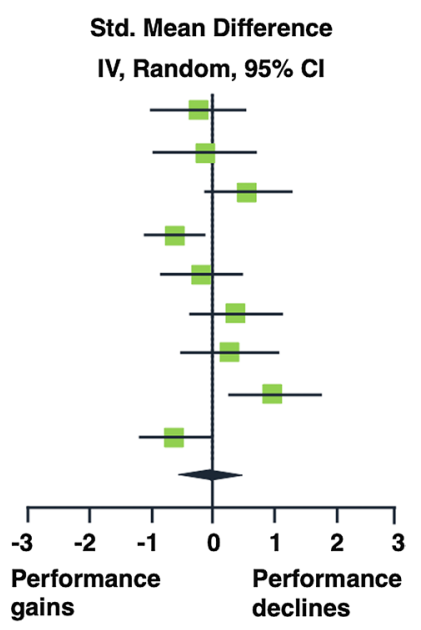

Fig. 3 Forest plot for the overall effect of aerobic exercise on subsequent measures of muscle power

large declines in muscle strength were found after $>30 \mathrm{~min}$ $\left(\mathrm{SMD}=1.02\right.$ [95\% CI 0.01-2.03], $p=0.049 ; I^{2}=47.4 \%$, four studies) while $\leq 30$ min of AE induced moderate decrements in muscle strength (SMD $=0.59$ [95\% CI 0.23-0.95], $p=0.013$; $I^{2}=0 \%$, four studies). Nevertheless, no statistically betweensubgroup differences were observed $(p=0.204)$. Running exercise resulted in small declines in muscle strength $(\mathrm{SMD}=0.28$ [95\% CI 0.08-0.47], $p=0.035 ; I^{2}=0 \%$, two studies) while cycling exercise produced moderate declines in muscle strength (SMD $=0.79$ [95\% CI 0.45-1.13], $p=0.002 ; I^{2}=0 \%$, five studies). Of note, the difference between subgroups was statistically significant $(p<0.0001)$.

\section{Discussion}

This is the first systematic review and meta-analysis to examine the acute effects of AE on measures of muscle strength and power in trained male individuals. The main findings of this systematic review with meta-analysis indicated that (1) AE resulted in acute moderate declines in subsequent (i.e., $\leq 15 \mathrm{~min}$ ) measures of lower limb muscle strength with no statistically significant effects on proxies of lower limb muscle power, (2) moderate-to-high intensity AE induced a moderate decline in muscle strength 
Table 3 Subgroup analysis for measures of muscle strength

\begin{tabular}{|c|c|c|c|c|c|c|}
\hline Subgroup & Studies $(N)$ & Participants $(N)$ & $\begin{array}{l}\text { Estimated effect } \\
\text { size Mean }(95 \% \\
\text { CI) }\end{array}$ & $\begin{array}{l}\text { Within-group } \\
p \text {-value }\end{array}$ & $\begin{array}{l}\text { Between sub- } \\
\text { group } p \text {-value }\end{array}$ & Effect descriptor \\
\hline \multicolumn{7}{|l|}{ AE intensity } \\
\hline Low & 2 & 16 & $\begin{array}{l}0.65(-1.45 \text { to } \\
2.75)\end{array}$ & 0.157 & 0.979 & \\
\hline $\begin{array}{l}\text { Moderate to } \\
\text { high }\end{array}$ & 5 & 53 & $\begin{array}{l}0.65(0.16- \\
1.13)\end{array}$ & 0.020 & & Medium \\
\hline \multicolumn{7}{|l|}{ AE type } \\
\hline Running & 2 & 18 & $\begin{array}{l}0.28(0.08- \\
0.47)\end{array}$ & 0.035 & $<0.001$ & Small \\
\hline Cycling & 5 & 51 & $\begin{array}{l}0.79(0.45- \\
1.13)\end{array}$ & 0.002 & & Medium \\
\hline \multicolumn{7}{|l|}{ AE duration } \\
\hline$\leq 30 \mathrm{~min}$ & 4 & 41 & $\begin{array}{l}0.59(0.23- \\
0.95)\end{array}$ & 0.013 & 0.204 & Medium \\
\hline$>30 \mathrm{~min}$ & 4 & 43 & $\begin{array}{l}1.02(0.01- \\
2.03)\end{array}$ & 0.049 & & Large \\
\hline
\end{tabular}

$A E$ aerobic exercise, $C I$ confidence interval

with no statistically significant effects of low-intensity AE, (3) AE lasting $>30$ min resulted in a large decline in muscle strength, whereas $\leq 30 \mathrm{~min}$ induced moderate declines, and (4) cycling exercise caused significantly larger decrements in muscle strength compared with running exercise.

\subsection{Main Effects of Aerobic Exercise on Subsequent Measures of Lower Limb Muscle Strength and Power}

The main effects of this study indicated that AE resulted in declines in muscle strength $(\mathrm{SMD}=0.79)$ but not power $(\mathrm{SMD}=0.04)$ in trained male individuals. In general, these findings are in agreement with the literature [34, 53]. Bentley et al. [53] studied the effects of AE until exhaustion on knee extensor IMVC and muscle activation in trained male athletes aged 25 years. They reported that $\mathrm{AE}$ resulted in significant IMVC declines immediately post-exercise $(\Delta 12 \%)$ and after $6 \mathrm{~h}(\Delta 6 \%)$ of rest. In addition, the authors showed that IMVC declines were related to reduced electromyographic activity and twitch torque, which is indicative of both central and peripheral mechanisms of neuromuscular fatigue [53]. Furthermore, Lattier et al. [34] examined the effects of high-intensity intermittent exercise (10 runs of 1 min each at $120 \%$ of maximal aerobic velocity) on subsequent knee extensor IMVC in well-trained male individuals aged 25 years. They reported an IMVC decline immediately $(\Delta 8 \%)$ and $65 \mathrm{~min}(\Delta 6 \%)$ after $\mathrm{AE}$. The same authors attributed the attenuation in IMVC to altered excitation-contraction coupling (decreased twitch contractile properties) and decreased maximal activation of the muscle. According to Lattier et al. [34], high-intensity intermittent exercise appears to induce both peripheral and central fatigue. In this context, the available literature is inconsistent. For example, Burnley and Jones [64] emphasized that the origin of neuromuscular fatigue depends on exercise intensity while Bishop [65] reported that fatigue following intermittent sprint exercise is primarily caused by peripheral mechanisms. Overall, there is ample evidence that AE has acute negative effects on measures of muscle strength [25, 66]. Of note, Lattier et al. [34] and Bentley et al. [53] reported that declines in IMVC last longer. Nevertheless, it should be highlighted that because of a lack of data, the quantitative analyses of this systematic review with meta-analysis solely considered measures of muscle strength within a time frame of $\leq 15 \mathrm{~min}$ after AE.

Unlike muscle strength, our findings indicated no significant effects of $\mathrm{AE}$ on subsequent measures of muscle power $(\mathrm{SMD}=0.04)$. In previous studies, effects of an allout $10-\mathrm{km}$ running competition on subsequent measures of muscle power (i.e., peak vertical jump power) were examined in male endurance athletes aged 18-26 years [54]. The main results indicated no changes in peak vertical jump power immediately after the $10-\mathrm{km}$ race. These authors speculated that motor unit recruitment during a $10-\mathrm{km}$ race does not involve fast-twitch muscle fibers, which are needed to produce high levels of muscle power [54]. Boullosa et al. [58] examined the effects of two endurance tasks performed until voluntary exhaustion (i.e., "Université de Montréal Track Test' ' and the time to exhaustion at maximal aerobic speed) on vertical jump performance (i.e., countermovement jump height) in well-trained male endurance runners aged 23 years. The authors reported an increase in vertical jump height 2 min after the two running protocols $(\Delta 13 \%$ and 
$\Delta 4 \%$, respectively), an observation attributed to PAPE [30]. Other studies have also shown AE-related PAPE on measures of muscle power [23, 27]. In this context, it is worth mentioning that PAPE is time dependent (up to $10 \mathrm{~min}$ ) but also moderated by the performance level of the respective athlete $[67,68]$. Furthermore, there are studies that reported a decline in vertical jump performance following $\mathrm{AE}[55$, 59]. For example, Johnston et al. [59] examined the effects of a maximum speed running session on vertical jump height in professional rugby players aged 21 years and found a significant decrease immediately after $\mathrm{AE}(\Delta 9 \%)$, which returned to baseline $2 \mathrm{~h}$ post-AE. Similar results were reported by Thomas et al. [55]. These authors [55] examined the effects of a simulated soccer match on measures of muscle power in professional players aged 21 years and reported a significant decrease in vertical jump height immediately after $(\Delta 13 \%)$, which lasted for up to $72 \mathrm{~h}(\Delta 5 \%)$. Taken together, these contradictory findings highlight the complex nature of neuromuscular fatigue. Indeed, our results suggest that neuromuscular fatigue is influenced by a large number of factors (e.g., individual training history or type of task applied).

Overall, our findings support the hypothesis that $\mathrm{AE}$ impairs subsequent measures of muscle strength [35, 57]. As to the underlying mechanisms, there is evidence that AE causes central (impaired neural drive) and peripheral (impaired excitation-contraction coupling) fatigue [34, 53] which ultimately results in muscle strength declines. Moreover, metabolic factors triggered by the AE seem to play a major role. Findings from different studies indicate that $\mathrm{AE}$ results in a decline in $\mathrm{Ca}^{2+}$ sensitivity, which may again inhibit the conversion of electrical stimuli to mechanical responses [69-71]. Results from animal and human studies [72] showed that exercise (e.g., cycling at $70 \%$ of $\mathrm{VO}_{2 \max }$ ) induces an increase in ryanodine receptor 1 phosphorylation that impairs myofibrillar $\mathrm{Ca}^{2+}$ processing and consequently muscle function. Additional factors such as metabolic acidosis (e.g., $\mathrm{pH}$ reduction and/or Pi accumulation) and impaired action potential (e.g., extracellular $\mathrm{K}^{+}$accumulation) appear to be fostered by AE $[69,71]$. Accordingly, it was reported that high AE volume increases extracellular $\mathrm{K}^{+}$accumulation [73, 74], altering sarcolemmal excitability [75]. Thereby, it seems that the above described physiological factors (e.g., muscle activation, defect in $\mathrm{Ca}^{2+}$ release, $\mathrm{Pi}$ accumulation) are moderated by AE modalities such as volume and intensity $[12,37]$.

Findings from this systematic review with meta-analysis are in line with the results from previous meta-analyses $[17,18]$ on chronic AE-SE sequencing effects. The existing data indicate that $\mathrm{SE}$ before $\mathrm{AE}$, but not $\mathrm{AE}$ before $\mathrm{SE}$, is more effective for improving muscle strength. In contrast, several studies [76-78] showed that high-intensity interval AE but also continuous AE performed prior to SE do not inhibit muscle protein synthesis. The gap between these findings may result from a multitude of possible CT modalities. Alternatively, muscular hypertrophy may not have been a major determinant of the training-induced strength improvements following SE-AE sequencing reported in previous studies $[17,18]$. Future studies should elucidate the underlying physiological mechanisms following different CT modalities. Further, our findings suggest that the capacity to generate maximal muscular power after AE is less affected compared with muscle strength. For instance, Latorre-Román et al. [24] examined the effects of running exercise (i.e., $4 \times 3 \times 400 \mathrm{~m}$ ) in trained runners and found no statistically significant change in vertical jump performance after AE. The production of muscle force can be regulated through motor unit recruitment, firing frequency, and synchronization [79]. Firing frequency appears to be a major mechanism that enables the muscle to rapidly generate force. Accordingly, firing frequency represents a major neural factor to regulate muscle power [80, 81]. Even though highly speculative, it can be argued that the applied AE protocols may have affected peripheral neuromuscular mechanisms rather than central mechanisms, which is why we found declines in muscle strength but not power. Given that this is a systematic review of the literature and not original research using electrophysiological testing, future studies are needed to verify this hypothesis.

\subsection{Subgroup Analyses}

Our results suggest that moderate-to-high intensity $(\mathrm{SMD}=0.68)$ but not low-intensity AE causes a decline in muscle strength. However, it should be mentioned that the observed differences between subgroups were not statistically significant and that only two studies were included that examined low-intensity training. Therefore, these results should be interpreted with caution. Our findings are in line with the available literature. For example, Lepers et al. [25] reported that male triathletes who cycled at an intensity of $75 \%$ of their maximal aerobic power (i.e., highest power in Watts across 2 min during a continuous incremental cycling test until exhaustion) experienced a 9\% decline in muscle strength. In addition, the same authors showed that cycling at $80-90 \%$ resulted in a $13 \%$ decline in maximal aerobic power. In terms of AE duration, our findings showed larger decrements for AE lasting $>30$ min compared with $\leq 30 \mathrm{~min}$ ( $\mathrm{SMD}=0.97$ vs $\mathrm{SMD}=0.72$, respectively). However, the difference was not statistically significant. Lepers et al. [35] investigated the effects of $2 \mathrm{~h}$ of cycling at $65 \%$ of maximal aerobic power in trained cyclists and found a significant reduction in maximal isometric force of the knee extensors $(\Delta 13 \%)$. In addition, Lattier et al. [34] investigated the effects of high-intensity uphill running (10 runs of 1 min each at $120 \%$ maximal aerobic velocity) 
in trained individuals and reported a significant decrease in isometric force of the knee extensors after $\operatorname{AE}(\Delta 8 \%)$. Regarding the underlying mechanisms, there is evidence that muscle glycogen depletion increases progressively, either with extended exercise duration and/or increased exercise intensity [82]. Moreover, earlier studies [35, 56, 57] reported an intensity-dependent and duration-dependent magnitude of peripheral and central fatigue.

The current findings suggest an AE-related effect that is specific to the applied AE type. More specifically, the results showed that cycling resulted in significantly larger decrements in muscle strength $(\mathrm{SMD}=0.79)$ compared with running exercise ( $\mathrm{SMD}=0.28$ ). There is evidence that cycling and running are associated with mitigated strength adaptations following CT compared with singlemode strength training [12]. However, cycling exercise has been associated with smaller interference effects compared with running exercise [83]. Amongst others, this can be attributed to eccentric muscle actions during running, which may increase muscle damage and therefore contribute to declines in measures of muscle strength and power [84]. Our results are contradictory to the existing literature and suggest that, for trained individuals, running exercise could be more appropriate to avoid neuromuscular fatigue and declines in muscle strength when AE is applied before SE. This may be partly explained by the role of cytoskeletal desmin and alpha-crystallin B proteins, which are known for their protection function of the myofiber integrity and cellular stabilization [85]. In fact, it was reported that particularly eccentric exercise stimuli induce an increase in alpha-crystallin B phosphorylation [86]. In consequence, we hypothesise that generally trained individuals may have higher total amounts of alpha-crystallin B, owing to training-induced skeletal muscle adaptation, which then, in turn, protects these individuals from skeletal muscle fatigue induced by eccentric exercise stimuli. The observed results can also be explained by methodological factors. While cycling exercise results in specific local fatigue with substrate depletion of the knee extensors, running exercise is a more general exercise type and involves larger muscle groups. Given that all included studies applied knee extensor strength tests after the AE protocol, it can be speculated that cycling compared with running exercise resulted in larger performance decrements due to more pronounced local muscular fatigue of the knee extensors. This is supported by Dingwell et al. [87], who reported significant local muscle fatigue in highly trained cyclists after cycling at $100 \%$ of $\mathrm{VO}_{2 \max }$ until voluntary exhaustion. The authors detected significantly lower electromyographic median frequencies immediately after the cycling exercise, most prominent in the biceps femoris and the gastrocnemius.

\section{Limitations}

This systematic review with meta-analysis has some limitations that warrant discussion. Above all, our results are valid for trained male adults. Thus, our findings cannot be translated to youth or female individuals. Another limitation is the heterogeneity of the endurance training protocols within the included studies. The various types of modalities, intensities, and durations demand different metabolic processes [88]. More specifically, intermittent vs continuous AE protocols may induce specific physiological adaptations. However, because of the limited number of available studies, protocol-specific analyses were not possible. Further, it can be anticipated that without an additional collection of physiological data and more structured modification of study designs and parameters (e.g., gene expression, cell signaling, blood markers of metabolism, and immunological stress) used in CT protocols concerning acute effects of $\mathrm{AE}$ on muscle strength and power, underlying (biological) mechanisms can hardly be identified. Studies involving trained individuals, which link performance with physiological data, are lacking and should be addressed in future studies. Moreover, the individual training status has a significant influence on shortterm and long-term response to a specific training stimulus [89]. Especially in the context of CT, it is a challenge to describe individual performance capabilities [39]. The training status described in CT studies can refer to endurance activity (e.g., cycling), strength or power activity (e.g., long-jump), or both (e.g., rugby). In general, the transferability of the results must be verified on an individual basis because the group of subjects examined within our work is rather heterogeneous.

\section{Conclusions}

The main findings of this systematic literature review with meta-analysis showed that AE results in significant and moderate declines in muscle strength but not power of involved muscles in trained male individuals. These results provide evidence of "acute interference" effects when AE is performed prior to SE. Further, our findings showed a negative influence of prior moderate-to-high intensity and longer duration $\mathrm{AE}$ on measures of muscle strength. Lowintensity and shorter duration AE did not mitigate strength performance. However, given the lack of statistically significant differences between subgroups (i.e., AE intensity [low vs moderate to high] and AE duration $[\leq 30 \mathrm{~min}$ vs $>30 \mathrm{~min}]$ ), it is not possible to conclude whether the acute AE effects on muscle strength were intensity and/ 
or duration dependent. Moreover, our results suggest that running compared with cycling exercise results in diminished acute negative effects in trained individuals. These findings may guide practitioners to better prescribe single training sessions in which trained individuals have to apply $\mathrm{AE}$ before SE because of environmental and/or infrastructural reasons (e.g., availability of training facilities). Further original research is needed with female individuals and youth athletes. Finally, future studies should assess objective physiological markers (e.g., gene expression, cell signalling, blood markers of metabolism, and immunological stress) in addition to performance measures to gain more insight into the physiological aspects of AE-induced acute performance declines in muscle strength.

Funding Open Access funding enabled and organized by Projekt DEAL. This study is part of the research project Resistance Training in Youth Athletes that was funded by the German Federal Institute of Sport Science (ZMVI4-081901/20-23).

\section{Declarations}

Conflict of interest Adrian Markov, Helmi Chaabene, Lukas Hauser, Sebastian Behm, Wilhelm Bloch, Christian Puta, and Urs Granacher declare that they have no conflicts of interest relevant to the content of this systematic review.

Availability of data and material The R script is available in Appendix $\mathrm{S} 1$ of the Electronic Supplementary Material [ESM]. Data from the included studies are available in the Appendix S2 of the ESM.

Code availability Not applicable.

Author contributions AM, HC, and UG made substantial contributions to conception and design; AM and SB contributed to data collection; AM and $\mathrm{HC}$ carried out the data analysis; AM, HC, LH, and UG interpreted the data; AM wrote the first draft of the manuscript and all authors were involved in revising it critically for important intellectual content; all authors provided the final approval of the version to be published and agreed to be accountable for all aspects of the work.

Open Access This article is licensed under a Creative Commons Attribution 4.0 International License, which permits use, sharing, adaptation, distribution and reproduction in any medium or format, as long as you give appropriate credit to the original author(s) and the source, provide a link to the Creative Commons licence, and indicate if changes were made. The images or other third party material in this article are included in the article's Creative Commons licence, unless indicated otherwise in a credit line to the material. If material is not included in the article's Creative Commons licence and your intended use is not permitted by statutory regulation or exceeds the permitted use, you will need to obtain permission directly from the copyright holder. To view a copy of this licence, visit http://creativecommons.org/licenses/by/4.0/.

\section{References}

1. Chaabene H, Negra Y, Capranica L, Prieske O, Granacher U. A needs analysis of karate kumite with recommendations for performance testing and training. Strength Cond J. 2019;41(3):3546. https://doi.org/10.1519/SSC.0000000000000445.

2. Kramer JF, Leger A, Paterson DH, Morrow A. Rowing performance and selected descriptive, field, and laboratory variables. Can J App Physiol. 1994;19(2):174-84. https://doi.org/10.1139/ h94-013.

3. Michael JS, Rooney KB, Smith R. The metabolic demands of kayaking: a review. J Sports Sci Med. 2008;7(1):1.

4. Baar K. Using molecular biology to maximize concurrent training. Sports Med. 2014;44(2):117-25.

5. Murach KA, Bagley JR. Skeletal muscle hypertrophy with concurrent exercise training: contrary evidence for an interference effect. Sports Med. 2016;46(8):1029-39. https://doi.org/10.1007/ s40279-016-0496-y.

6. Gäbler M, Prieske O, Hortobágyi T, Granacher U. The effects of concurrent strength and endurance training on physical fitness and athletic performance in youth: a systematic review and meta-analysis. Front Physiol. 2018;9:1057. https://doi.org/10. 3389/fphys.2018.01057.

7. Hickson RC. Interference of strength development by simultaneously training for strength and endurance. Eur J Appl Physiol Occup Physiol. 1980;45(2-3):255-63.

8. Izquierdo M, Häkkinen K, Ibanez J, Kraemer WJ, Gorostiaga EM. Effects of combined resistance and cardiovascular training on strength, power, muscle cross-sectional area, and endurance markers in middle-aged men. Eur J Appl Physiol. 2005;94(1-2):70-5.

9. Karavirta L, Häkkinen A, Sillanpää E, García-López D, Kauhanen A, Haapasaari A, et al. Effects of combined endurance and strength training on muscle strength, power and hypertrophy in 40-67-year-old men. Scand J Med Sci Sports. 2011;21(3):402-11. https://doi.org/10.1111/j.1600-0838.2009.01059.x.

10. Schumann M, Yli-Peltola K, Abbiss CR, Häkkinen K. Cardiorespiratory adaptations during concurrent aerobic and strength training in men and women. PLoS ONE. 2015;10(9):139279.

11. Docherty D, Sporer B. A proposed model for examining the interference phenomenon between concurrent aerobic and strength training. Sports Med. 2000;30(6):385-94.

12. Fyfe JJ, Bishop DJ, Stepto NK. Interference between concurrent resistance and endurance exercise: molecular bases and the role of individual training variables. Sports Med. 2014;44(6):743-62. https://doi.org/10.1007/s40279-014-0162-1.

13. Vechin FC, Conceição MS, Telles GD, Libardi CA, Ugrinowitsch C. Interference phenomenon with concurrent strength and high-intensity interval training-based aerobic training: an updated model. Sports Med. 2021;51(4):599-605.

14. Jones TW, Howatson G, Russell M, French DN. Performance and neuromuscular adaptations following differing ratios of concurrent strength and endurance training. J Strength Cond Res. 2013;27(12):3342-51. https://doi.org/10.1519/JSC.0b013e3181 b2cf39.

15. Kraemer WJ, Patton JF, Gordon SE, Harman EA, Deschenes MR, Reynolds K, et al. Compatibility of high-intensity strength and endurance training on hormonal and skeletal muscle adaptations. J Appl Physiol. 1995;78(3):976-89.

16. Fett D, Trompeter K, Platen P. Back pain in elite sports: a cross-sectional study on 1114 athletes. PLoS ONE. 2017;12(6): e0180130. https://doi.org/10.1152/jappl.1995.78.3.976.

17. Murlasits Z, Kneffel Z, Thalib L. The physiological effects of concurrent strength and endurance training sequence: a systematic review and meta-analysis. J Sports Sci. 2018;36(11):1212-9. https://doi.org/10.1080/02640414.2017.1364405.

18. Eddens L, van Someren K, Howatson G. The role of intra-session exercise sequence in the interference effect: a systematic review with meta-analysis. Sports Med. 2018;48(1):177-88. https://doi. org/10.1007/s40279-017-0784-1. 
19. de Araújo Ruas VD, Figueira TR, Denadai BS, Greco CC. Effect of cycling exercise at different pedal cadences on subsequent muscle strength. J Exerc Sci Fit. 2011;9(2):93-9. https://doi.org/10. 1016/S1728-869X(12)60004-5.

20. Schumann M, Walker S, Izquierdo M, Newton RU, Kraemer WJ, Häkkinen K. The order effect of combined endurance and strength loadings on force and hormone responses: effects of prolonged training. Eur J Appl Physiol. 2014;114(4):867-80.

21. Taipale RS, Schumann M, Mikkola J, Nyman K, Kyröläinen H, Nummela A, et al. Acute neuromuscular and metabolic responses to combined strength and endurance loadings: the "order effect" in recreationally endurance trained runners. J Sports Sci. 2014;32(12):1155-64. https://doi.org/10.1080/02640414.2014. 889842.

22. Boullosa DA, Tuimil JL, Alegre LM, Iglesias E, Lusquiños F. Concurrent fatigue and potentiation in endurance athletes. Int $\mathrm{J}$ Sports Physiol Perf. 2011;6(1):82-93. https://doi.org/10.1123/ ijspp.6.1.82.

23. García-Pinillos F, Soto-Hermoso VM, Latorre-Román PA. Acute effects of extended interval training on countermovement jump and handgrip strength performance in endurance athletes: postactivation potentiation. J Strength Cond Res. 2015;29(1):11-21. https://doi.org/10.1519/JSC.0000000000000591.

24. Latorre-Román PÁ, García-Pinillos F, Martínez-López EJ, SotoHermoso VM. Concurrent fatigue and postactivation potentiation during extended interval training in long-distance runners. Motriz Revista de Educação Física. 2014;20(4):423-30. https://doi.org/ 10.1590/S1980-65742014000400009.

25. Lepers R, Theurel J, Hausswirth C, Bernard T. Neuromuscular fatigue following constant versus variable-intensity endurance cycling in triathletes. J Sci Med in Sport. 2008;11(4):381-9. https://doi.org/10.1016/j.jsams.2007.03.001.

26. Wilhelm EN, Radaelli R, Perin D, Cunha GS, Cadore EL, Laitano $\mathrm{O}$, et al. The influence of running and cycling on subsequent maximal muscular performance. Isokinet Exerc Sci. 2014;22(2):11522. https://doi.org/10.3233/IES-130527.

27. Vuorimaa T, Virlander R, Kurkilahti P, Vasankari T, Häkkinen K. Acute changes in muscle activation and leg extension performance after different running exercises in elite long distance runners. Eur J Appl Physiol. 2006;96(3):282-91.

28. Fitts RH. Cellular mechanisms of muscle fatigue. Physiol Rev. 1994;74(1):49-94. https://doi.org/10.1152/physrev.1994.74.1.49.

29. Gandevia SC. Spinal and supraspinal factors in human muscle fatigue. Physiol Rev. 2001;81(4):1725-89. https://doi.org/10. 1152/physrev.2001.81.4.1725.

30. Prieske O, Behrens M, Chaabene H, Granacher U, Maffiuletti NA. Time to differentiate postactivation "potentiation" from "performance enhancement" in the strength and conditioning community. Sports Med. 2020;50:1559-65. https://doi.org/10.1007/ s40279-020-01300-0.

31. Enoka RM, Duchateau J. Translating fatigue to human performance. Med Sci Sports Exerc. 2016;48(11):2228. https://doi.org/ 10.1249/2FMSS.0000000000000929.

32. Noakes TDO. Fatigue is a brain-derived emotion that regulates the exercise behavior to ensure the protection of whole body homeostasis. Front Physiol. 2012;3:82. https://doi.org/10.3389/fphys. 2012.00082

33. Weir J, Beck T, Cramer J, Housh T. Is fatigue all in your head? A critical review of the central governor model. Br J Sports Med. 2006;40(7):573-86. https://doi.org/10.1136/bjsm.2005.023028.

34. Lattier G, Millet G, Martin A, Martin V. Fatigue and recovery after high-intensity exercise. Part II: recovery interventions. Int J Sports Med. 2004;25(7):509-15.
35. Lepers R, Hausswirth C, Maffiuletti N, Brisswalter J, Van Hoecke J. Evidence of neuromuscular fatigue after prolonged cycling exercise. Med Sci Sports Exerc. 2000;32(11):1880-6.

36. Coffey VG, Hawley JA. Concurrent exercise training: do opposites distract? J Physiol. 2017;595(9):2883-96. https://doi.org/10.1113/ JP272270.

37. Bishop DJ, Bartlett J, Fyfe J, Lee M. Methodological considerations for concurrent training.Concurrent aerobic and strength training. Springer, Cham, Switzerland, 2019: p. 183-96.

38. Liberati A, Altman DG, Tetzlaff J, Mulrow C, Gøtzsche PC, Ioannidis JP, et al. The PRISMA statement for reporting systematic reviews and meta-analyses of studies that evaluate health care interventions: explanation and elaboration. J Clin Epidemiol. 2009;62(10):e1-34. https://doi.org/10.1016/j.jclinepi.2009.06.006.

39. Fyfe JJ, Loenneke JP. Interpreting adaptation to concurrent compared with single-mode exercise training: some methodological considerations. Sports Med. 2018;48(2):289-97. https://doi.org/ 10.1007/s40279-017-0812-1.

40. MacMahon C, Parrington L. Not all athletes are equal, but don't call me an exerciser: response to Araujo and Scharhag. Scand J Med Sci Sports. 2017;27:904-6. https://doi.org/10.1111/sms. 12864.

41. Wenger HA, Bell GJ. The interactions of intensity, frequency and duration of exercise training in altering cardiorespiratory fitness. Sports Med. 1986;3(5):346-56.

42. Digitizer IP. How to extract data from graphs using plot digitizer or getdata graph digitizer. 2020. http://getdata-graph-digitizer. com/.

43. Wojtyniak JG, Britz H, Selzer D, Schwab M, Lehr T. Data digitizing: accurate and precise data extraction for quantitative systems pharmacology and physiologically-based pharmacokinetic modeling. CPT Pharmacometr Syst Pharmacol. 2020;9(6):322-31. https://doi.org/10.1002/psp4.12511.

44. Galna B, Peters A, Murphy AT, Morris ME. Obstacle crossing deficits in older adults: a systematic review. Gait Posture. 2009;30(3):270-5. https://doi.org/10.1016/j.gaitpost.2009.05.022.

45. Sidik K, Jonkman JN. A comparison of heterogeneity variance estimators in combining results of studies. Stat Med. 2007;26(9):1964-81. https://doi.org/10.1002/sim.2688

46. Balduzzi S, Rücker G, Schwarzer G. How to perform a metaanalysis with R: a practical tutorial. Evid Based Ment Health. 2019;22(4):153-60. https://doi.org/10.1136/ebmental-2019-30011 7.

47. Viechtbauer $\mathrm{W}$. Conducting meta-analyses in $\mathrm{R}$ with the metafor package. J Stat Softw. 2010;36(3):1-48. https://doi.org/10.18637/ jss.v036.i03.

48. Garber CE, Blissmer B, Deschenes MR, Franklin BA, Lamonte MJ, Lee I-M, et al. American College of Sports Medicine position stand. Quantity and quality of exercise for developing and maintaining cardiorespiratory, musculoskeletal, and neuromotor fitness in apparently healthy adults: guidance for prescribing exercise. In: Medicine and science in sports and exercise 2011;43(7), S. 1334-1359. https://doi.org/10.1249/MSS.0b013e318213fefb.

49. Moritani T, Nagata A, Devries HA, Muro M. Critical power as a measure of physical work capacity and anaerobic threshold. Ergonomics. 1981;24(5):339-50. https://doi.org/10.1080/0014013810 8924856.

50. Cohen J. Statistical power analysis for the behavioral sciences. 2nd ed. Hillsdale: Lawrence Erlbaum Associates Inc.; 1988. p. 13.

51. Higgins JP, Thompson SG, Deeks JJ, Altman DG. Measuring inconsistency in meta-analyses. BMJ. 2003;327(7414):557-60. https://doi.org/10.1136/bmj.327.7414.557.

52. Team RC. R: A language and environment for statistical computing. 2013. 
53. Bentley DJ, Smith PA, Davie AJ, Zhou S. Muscle activation of the knee extensors following high intensity endurance exercise in cyclists. Eur J Appl Physiol. 2000;81(4):297-302.

54. Gómez AL, Radzwich RJ, Denegar CR, Volek JS, Rubin MR, Bush JA, et al. The effects of a 10-kilometer run on muscle strength and power. J Strength Cond Res. 2002;16(2):184-91.

55. Thomas K, Dent J, Howatson G, Goodall S. Etiology and recovery of neuromuscular fatigue following simulated soccer match-play. Med Sci Sports Exerc. 2017;49(5):955-64.

56. Thomas K, Elmeua M, Howatson G, Goodall S. Intensity-dependent contribution of neuromuscular fatigue after constant-load cycling. Med Sci Sports Exerc. 2016;48(9):1751-60.

57. Thomas K, Goodall S, Stone M, Howatson G, Gibson ASC, Ansley L. Central and peripheral fatigue in male cyclists after 4-, 20-, and 40-km time trials. Med Sci Sports Exerc. 2015;47(3):537-46.

58. Boullosa DA, Tuimil JL. Postactivation potentiation in distance runners after two different field running protocols. J Strength Cond Res. 2009;23(5):1560-5. https://doi.org/10.1519/JSC.0b013 e3181a3ce61.

59. Johnston MJ, Cook CJ, Drake D, Costley L, Johnston JP, Kilduff LP. The neuromuscular, biochemical, and endocrine responses to a single-session vs double-session training day in elite athletes. J Strength Cond Res. 2016;30(11):3098-106. https://doi.org/10. 1519/JSC.0000000000001423.

60. Sparkes W, Turner A, Cook C, Weston M, Russell M, Johnston $\mathrm{M}$, et al. The neuromuscular, endocrine and mood responses to a single versus double training session day in soccer players. J Sci Med Sport. 2020;23(1):69-74. https://doi.org/10.1016/j.jsams. 2019.08.291.

61. Sparkes W, Turner AN, Weston M, Russell M, Johnston M, Kilduff LP. The effect of training order on neuromuscular, endocrine and mood response to small-sided games and resistance training sessions over a 24-h period. J Sci Med Sport. 2020;23(9):866-71. https://doi.org/10.1016/j.jsams.2020.01.017.

62. García-Pinillos F, Molina-Molina A, Latorre-Román PÁ. Impact of an incremental running test on jumping kinematics in endurance runners: can jumping kinematic explain the post-activation potentiation phenomenon? Sports Biomech. 2016;15(2):103-15. https://doi.org/10.1080/14763141.2016.1158860.

63. Panissa VL, Cal Abad CC, Julio UF, Andreato LV, Franchini E. High-intensity intermittent exercise and its effects on heart rate variability and subsequent strength performance. Front Physiol. 2016;7:81. https://doi.org/10.3389/fphys.2016.00081.

64. Burnley M, Jones AM. Power-duration relationship: physiology, fatigue, and the limits of human performance. Eur J Sport Sci. 2018;18(1):1-12. https://doi.org/10.1080/17461391.2016.12495 24.

65. Bishop DJ. Fatigue during intermittent-sprint exercise. Clin Exp Pharmacol Physiol. 2012;39(9):836-41. https://doi.org/10.1111/j. 1440-1681.2012.05735.x

66. Bentley DJ, Zhou S, Davie AJ. The effect of endurance exercise on muscle force generating capacity of the lower limbs. J Sci Med Sport. 1998;1(3):179-88. https://doi.org/10.1016/S1440-2440(98) 80013-3.

67. Wilson JM, Duncan NM, Marin PJ, Brown LE, Loenneke JP, Wilson SM, et al. Meta-analysis of postactivation potentiation and power: effects of conditioning activity, volume, gender, rest periods, and training status. J Strength Cond Res. 2013;27(3):854-9. https://doi.org/10.1519/JSC.0b013e31825c2bdb.

68. Seitz LB, Haff GG. Factors modulating post-activation potentiation of jump, sprint, throw, and upper-body ballistic performances: a systematic review with meta-analysis. Sports Med. 2016;46(2):231-40. https://doi.org/10.1007/s40279-015-0415-7 .

69. Fitts R. The role of acidosis in fatigue: pro perspective. Med Sci Sports Exerc. 2016;48(11):2335-8.
70. Gehlert S, Bloch W, Suhr F. Ca2+-dependent regulations and signaling in skeletal muscle: from electro-mechanical coupling to adaptation. Int J Mol Sci. 2015;16(1):1066-95. https://doi.org/ 10.3390/ijms16011066.

71. Place N, Yamada T, Bruton JD, Westerblad H. Muscle fatigue: from observations in humans to underlying mechanisms studied in intact single muscle fibres. Eur J Appl Physiol. 2010;110(1):1-15.

72. Bellinger AM, Reiken S, Dura M, Murphy PW, Deng S-X, Landry DW, et al. Remodeling of ryanodine receptor complex causes "leaky" channels: a molecular mechanism for decreased exercise capacity. Proc Natl Acad Sci USA. 2008;105(6):2198-202. https:// doi.org/10.1073/pnas.0711074105.

73. Overgaard K, Lindstrøm T, Ingemann-Hansen T, Clausen T. Membrane leakage and increased content of $\mathrm{Na}+\mathrm{K}+$ pumps and $\mathrm{Ca} 2+$ in human muscle after a $100-\mathrm{km}$ run. J Appl Physiol. 2002;92(5):1891-8. https://doi.org/10.1152/japplphysiol.00669. 2001.

74. Pastene J, Germain M, Allevard A, Gharib C, Lacour J. Water balance during and after marathon running. Eur J Appl Physiol Occup Physiol. 1996;73(1):49-55.

75. Fitts RH. Muscle fatigue: the cellular aspects. Am J Sports Med. 1996;24(6_suppl):S9-13.

76. Fyfe JJ, Bishop DJ, Zacharewicz E, Russell AP, Stepto NK. Concurrent exercise incorporating high-intensity interval or continuous training modulates mTORC1 signaling and microRNA expression in human skeletal muscle. Am J Physiol Regul Integr Comp Physiol. 2016;310(11):R1297-311. https://doi.org/10.1152/ ajpregu.00479.2015.

77. Apro W, Moberg M, Hamilton DL, Ekblom B, van Hall G, Holmberg HC, Blomstrand E. Resistance exercise-induced S6K1 kinase activity is not inhibited in human skeletal muscle despite prior activation of AMPK by high-intensity interval cycling. Am J Physiol Endocrinol Metabol. 2015;308(6):E470-81. https://doi. org/10.1152/ajpendo.00486.2014.

78. Lundberg TR, Fernandez-Gonzalo R, Tesch PA. Exerciseinduced AMPK activation does not interfere with muscle hypertrophy in response to resistance training in men. J Appl Physiol. 2014;116(6):611-20. https://doi.org/10.1152/japplphysiol.01082. 2013.

79. Cormie P, McGuigan MR, Newton RU. Developing maximal neuromuscular power. Sports Med. 2011;41(1):17-38.

80. Enoka RM. Morphological features and activation patterns of motor units. J Clin Neurophysiol. 1995;12(6):538-59. https://doi. org/10.1097/00004691-199511000-00002.

81. Zehr EP, Sale DG. Ballistic movement: muscle activation and neuromuscular adaptation. Can J Appl Physiol. 1994;19(4):363-78. https://doi.org/10.1139/h94-030.

82. Gollnick P, Piehl K, Saltin B. Selective glycogen depletion pattern in human muscle fibres after exercise of varying intensity and at varying pedalling rates. J Physiol. 1974;241(1):45-57. https://doi. org/10.1113/jphysiol.1974.sp010639.

83. Wilson JM, Marin PJ, Rhea MR, Wilson SMC, Loenneke JP, Anderson JC. Concurrent training: a meta-analysis examining interference of aerobic and resistance exercises. J Strength Cond Res. 2012;26(8):2293-307. https://doi.org/10.1519/JSC.0b013 e31823a3e2d.

84. Gergley JC. Comparison of two lower-body modes of endurance training on lower-body strength development while concurrently training. J Strength Cond Res. 2009;23(3):979-87. https://doi.org/ 10.1519/JSC.0b013e3181a0629d.

85. Jacko D, Bersiner K, Schulz O, Przyklenk A, Spahiu F, Höhfeld J, et al. Coordinated alpha-crystallin B phosphorylation and desmin expression indicate adaptation and deadaptation to resistance exercise-induced loading in human skeletal muscle. Am J Physiol Cell Physiol. 2020;319(2):C300-12. 
86. Frankenberg NT, Lamb GD, Overgaard K, Murphy RM, Vissing K. Small heat shock proteins translocate to the cytoskeleton in human skeletal muscle following eccentric exercise independently of phosphorylation. J Appl Physiol. 2014;116(11):1463-72. https://doi.org/10.1152/japplphysiol.01026.2013.

87. Dingwell JB, Joubert JE, Diefenthaeler F, Trinity JD. Changes in muscle activity and kinematics of highly trained cyclists during fatigue. IEEE Trans Biomed Eng. 2008;55(11):2666-74. https:// doi.org/10.1109/TBME.2008.2001130.
88. Ferretti G, Bringard A, Perini R. An analysis of performance in human locomotion. Eur J Appl Physiol. 2011;111(3):391-401. https://doi.org/10.1007/s00421-010-1482-y.

89. Camera DM, Smiles WJ, Hawley JA. Exercise-induced skeletal muscle signaling pathways and human athletic performance. Free Radical Biol Med. 2016;98:131-43. https://doi.org/10.1016/j.freer adbiomed.2016.02.007.

\section{Authors and Affiliations}

\section{Adrian Markov ${ }^{1} \cdot$ Helmi Chaabene ${ }^{4} \cdot$ Lukas Hauser $^{1} \cdot$ Sebastian Behm ${ }^{1} \cdot$ Wilhelm Bloch $^{2} \cdot$ Christian Puta $^{3}$. Urs Granacher $^{1} \mathbb{B}$}

1 Division of Training and Movement Sciences, Research Focus Cognition Sciences, Faculty of Human Sciences, University of Potsdam, Am Neuen Palais 10, Bldg. 12, 14469 Potsdam, Germany

2 Department of Molecular and Cellular Sport Medicine, German Sport University, Cologne, Germany
3 Department of Sports Medicine and Health Promotion, Friedrich-Schiller-University Jena, Jena, Germany

4 Faculty of Human Sciences, University of Potsdam, Potsdam, Germany 